\title{
EVALUACIÓN DEL COSTO Y RENDIMIENTO DEL MANEJO BAJO UN CRITERIO SILVOPASTORAL DE UNA PLANTACIÓN DE Pinus ponderosa Douglas ex Lawson EN COYHAIQUE, REGIÓN DE AYSÉN, CHILE
}

\author{
Salinas, Jaime ${ }^{4}$; Inostroza, Patricio ${ }^{5}$ y Acuña, Bernardo 6
}

\section{RESUMEN}

En la Región de Aysén, existen 35.671 hectáreas cubiertas con plantaciones forestales. La especie principal en la región es pino ponderosa (Pinus ponderosa), le siguen en importancia pino contorta (Pinus contorta) y pino oregón (Pseudotsuga menziesii), especies adaptadas a condiciones de bajas temperaturas y presencia de nieve en forma temporal. La introducción de estas plantaciones se inicia en la década del 70, buscando revertir los procesos erosivos ocasionados por grandes incendios forestales ocurridos principalmente durante la primera mitad del siglo pasado, que comprometieron cerca de 3,5 millones de hectáreas en la región.

En la actualidad una proporción de estas superficies forestadas no están bajo manejo silvícola debido a diversas razones, entre las que se cuentan la falta de incentivos al manejo, el escaso conocimiento que existía sobre las propiedades de sus maderas, la competencia con maderas procedentes del norte, el alto costo de cosecha y otras.

El presente trabajo analizó el costo y rendimiento de una faena de raleo bajo un criterio de manejo silvopastoral en una plantación de 18 años de $P$. ponderosa ubicada en la comuna de Coyhaique, Región de Aysén de Chile. Se realizó un estudio de tiempo y rendimiento de las principales actividades asociadas al sistema de cosecha forestal tradicional (no mecanizado), para determinar productividad y costos de la faena y generar información para futuros instrumentos de fomentos de la actividad de manejo de plantaciones forestales.

Con la aplicación de un raleo por lo bajo a una intensidad del $50 \%$ de la densidad, se logró obtener el costo de manejo de la plantación, el cual fue de M\$963.7/ha. Por otro lado, la productividad obtenida posterior a la intervención fue de 246 "/ha, 464 postes/ha (2,2 m de largo) y $40 \mathrm{~m}^{3} / \mathrm{ha}$ de leña. Con la valorización de estos productos obtenidos del raleo fue posible obtener M\$ 1.399/ha, cubriendo el costo de cosecha y obteniendo una utilidad de $M \$ 439 /$ ha bajo las características de la plantación estudiada.

Palabras claves: Raleo, estudio de tiempo y rendimiento, agroforestería.

\footnotetext{
${ }^{4}$ Ingeniero Forestal, Instituto Forestal, sede Patagonia, Coyhaique, Chile. jsalinas@infor.cl

${ }^{5}$ Ingeniero Forestal, Consultor privado, Coyhaique, Chile.

${ }^{6}$ Instituto Forestal, sede Patagonia, Coyhaique, Chile.
} 


\section{SUMMARY}

A total of 35,671 ha are covered with forest plantations in the Aysén Region. The most important species in the region is Ponderosa Pine (Pinus ponderosa), the second one is Lodgepole Pine (Pinus contorta) and finally Douglas Fir (Pseudotsuga menziesii). These are species adapted to low temperatures conditions and temporarily snow presence. By the 70 s of the past century began the planted forests establishment to reverse the erosive processes caused by large forest fires registered mainly during the century first half, which affected about 3.5 million hectares in the region.

Nowadays, a proportion of these forested areas are not under silvilculture management because of several reasons, such as the lack of incentives to silviculture treatments, the insufficient knowledge on their wood properties, the usual use of woods coming from the north, the high harvesting costs and others.

This study analyzed the cost and yield of a thinning intervention in a 18 years old Pinus ponderosa plantation under silvopastoral management criterion, located at the Coyhaique commune. A time and yield study on the main activities associated to the traditional forest harvesting system (no mechanized) was carried out to determine the intervention's productivity and costs and to generate information for future incentives to promote plantations management.

Through a $50 \%$ intensity thinning was obtained the plantation management cost of $\mathrm{M} \$$ 963.7/ha. The productivity was 246 "/ha for sawn wood, 464 poles/ha ( $2.2 \mathrm{~m}$ length) and $40 \mathrm{~m}^{3} / \mathrm{ha}$ of firewood. These products represent a $M \$ 1,399 /$ ha income and a $M \$ 439 /$ ha net gain under the studied plantation characteristics.

Keywords: Thinning, time and yield study, agroforestry. 


\section{INTRODUCCIÓN}

El desarrollo del sector forestal chileno lo ha llevado a constituirse en una de las principales actividades económicas del país, aportando al PIB un monto cercano al 2,0\%. La cubierta forestal del país es de 17 millones de hectáreas, $22,5 \%$ de la superficie nacional continental, y está constituida por 14,6 millones de hectáreas de bosques nativos $(86 \%)$ y 2,4 millones de hectáreas de plantaciones forestales (14\%) (INFOR, 2018).

A inicios de los años 70 del siglo pasado la superficie de plantaciones era de unas 450 mil hectáreas y desde entonces el desarrollo de la industria forestal y los incentivos estatales a la forestación (DL. № 701 de 1974, vigente hasta 2012) han mantenido una tasa de plantación anual cercana a la 100.000 ha en promedio, la que descontados los consumos anuales ha llevado el patrimonio de plantaciones en pie a diciembre de 2016 a 2,4 millones de hectáreas.

Inicialmente las plantaciones eran muy mayoritariamente de pino radiata (Pinus radiata), posteriormente se ha incrementado la participación de eucaliptos (Eucalyptus globulus y Eucalyptus nitens) y otras especies coníferas y latifoliadas. A diciembre 2016 las plantaciones están constituidas por 1.391 .039 ha de pino radiata, 592.136 ha de Eucalyptus globulus, 268.181 ha de Eucalyptus nitens y 162.852 de otras especies (INFOR, 2018).

A diciembre de 2016 en la Región de Aysén existen 35.671 ha de plantaciones en pie, principalmente de pino ponderosa (Pinus ponderosa) con 19.348 ha, le siguen en importancia pino contorta (Pinus contorta) y pino oregón (Pseudotsuga menziesii), y superficies menores de otras especies (INFOR, 2018).

Esta extensa región del país cuenta con la mayor superficie de bosques nativos, con 4,8 millones de hectáreas que representan un tercio de la superficie de bosques nativos a nivel nacional y $44,9 \%$ de la superficie regional (INFOR, 2018). No obstante, la crianza de ganado es una tradición regional desde los tiempos de la colonización de estas tierras, así el uso de los suelos productivos agropecuarios, 746.807 ha, está en un $88,7 \%$ destinado a la producción ganadera (INE, 1997).

El objetivo inicial de las plantaciones forestales en la región fue revertir los procesos erosivos de los suelos (Salinas y Acuña, 2013) originados como resultado de los grandes incendios forestales en el periodo de colonización de esta región austral, que comprometieron cerca de 3,5 millones de hectáreas (Quintanilla, 2008) y dejaron los suelos desprovistos de vegetación, deteriorando seriamente su capacidad productiva.

Del total de superficie forestada en la región, aproximadamente el $33 \%$ se encuentra en manos de pequeños y medianos propietarios forestales, los que a través de los instrumentos de fomento del Estado (DL № 701 de 1974) recibieron incentivos por hasta el $90 \%$ de los costos netos de sus forestaciones.

El mencionado cuerpo legal contemplaba también incentivos estatales para el manejo de las plantaciones, pero lamentablemente estos fueron muy parcialmente empleados dado que este instrumento de fomento entregaba los incentivos al manejo para plantaciones de hasta 10 años de edad, condición que dejaba al margen las plantaciones de la región debido a que estas por sus condiciones de crecimiento no reunían aún las características técnicas para ser sometidas a prácticas de manejo forestal a esa edad.

Las plantaciones forestales de la región no son actualmente lo suficientemente atractivas para el manejo forestal, debido a múltiples factores que han impedido su desarrollo, como altos costos de cosecha, insuficiente conocimiento de las propiedades de la madera, competencia con maderas traídas del norte, inexistencia de instrumentos de fomento en la actualidad, reducido mercado local, y otros.

Esta situación ha desmotivado a los productores regionales a invertir en su manejo, sin embargo se observa un potencial interesante para el uso silvopastoral que podría incentivar los futuros manejos. 


\section{OBJETIVOS}

En el país existe escasa documentación sobre intervenciones tardías en plantaciones de rápido crecimiento, por lo cual este trabajo se plantea como objetivo generar información sobre costos y rendimientos en una faenas de manejo silvopastoral en una plantación de pino ponderosa de pequeños propietarios de la Región de Aysén.

\section{METODOLOGÍA}

\section{Área de Estudio}

Los trabajos se realizaron en un área ubicada en la localidad de Emperador Guillermo (Figura № 1), distante $36 \mathrm{~km}$ al noroeste de la ciudad de Coyhaique, en un predio denominado La Amistad $\left(45^{\circ} 18,5^{\prime} \mathrm{S}, 72^{\circ} 4,9^{\prime} \mathrm{O}\right)$. El sector posee una geografía montañosa con valles angostos moldeados principalmente por la actividad glacial. Esta zona corresponde a la ecorregión Templada Húmeda Intermedia ( $\mathrm{Cfb}$ ), los suelos predominantes son andisoles), el clima es frío lluvioso con temperaturas y precipitaciones medias de $8,1 \stackrel{\circ}{ } \mathrm{C}$ y $1.149 \mathrm{~mm} / \mathrm{año}$, respectivamente (Hepp y Stolpe, 2014). La topografía del entorno se caracteriza por lomajes suaves. La vegetación de la zona está compuesta por bosques puros de lenga (Nothofagus pumilio) en el límite altitudinal, en laderas de menor altitud ocurren bosques mixtos bajos de notro (Embothrium coccineum) y coigue (Nothofagus dombeyi).

En el predio se ubica una plantación de pino ponderosa de 18 años de edad, con una densidad de 1.360 arb/ha, un área basal de $53,25 \mathrm{~m}^{2} /$ ha y una altura promedio de $10,8 \mathrm{~m}$. El sotobosque es escaso, compuesto principalmente por rosa mosqueta (Rosa rubigenosa) y cubierto por acículas de pino. Se eligió un sector de 0,25 ha y en el mes de marzo del 2017 se aplicó un raleo con criterio silvopastoral a una intensidad del $50 \%$ de la densidad.

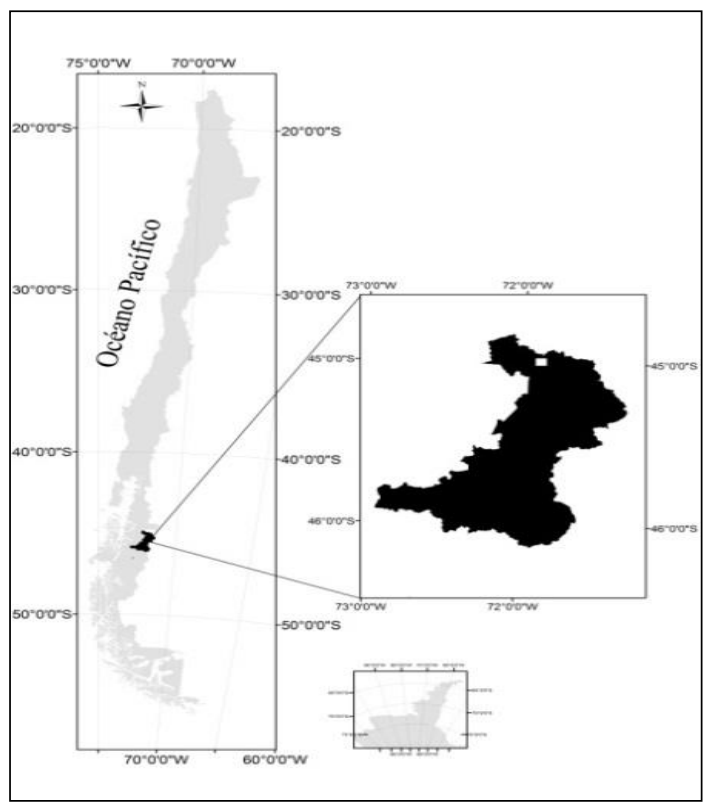

Figura $\mathrm{N}^{\circ} 1$

UBICACIÓN DEL ENSAYO 


\section{Inventario Forestal}

La caracterización de la estructura forestal del rodal, antes y después del raleo, se realizó a través de un inventario forestal compuesto por 3 parcelas circulares de $250 \mathrm{~m}^{2}$ (radio=8,92 $\mathrm{m}$ ). Los parámetros evaluados fueron diámetro del árbol a $1,3 \mathrm{~m}$ del suelo (DAP) y altura total de árboles $(\mathrm{Ht})$. Se determinaron valores de densidad (arb/ha), área basal $\left(\mathrm{m}^{2} / \mathrm{ha}\right)$ y para la estimación del volumen ( $\mathrm{m}^{3} / \mathrm{ha}$ ) se utilizó la ecuación propuesta por Gonda (2001).

$$
\begin{aligned}
\mathrm{V}=0,000214+0,000030 * & \mathrm{DAP}^{2}{ }^{*} \mathrm{Ht}+0,000538 * \text { DAP } \\
\text { Donde: } & \begin{array}{ll}
\mathrm{V} & =\text { Volumen total sin corteza }\left(\mathrm{m}^{3}\right) \\
\text { DAP } & =\text { Diámetro a la altura del pecho }(\mathrm{cm}) \\
\mathrm{Ht} & =\text { Altura total }(\mathrm{m})
\end{array}
\end{aligned}
$$

\section{Sistema de Explotación Forestal}

El sistema de explotación utilizado corresponde al de una faena forestal tradicional de pequeños propietarios de la Región de Aysén, que en el caso particular de este estudio estuvo compuesta por la siguiente secuencia de actividades o subsistemas:

Subsistema Volteo: Esta actividad fue realizada por un operador especializado, se ejecutó en forma manual mediante el uso de una motosierra marca STIHL, modelo 250 de 3,1 Hp con espada de 18" de largo. Esta actividad consideró las etapas de selección de árbol a cortar, limpieza de vías de escape, despeje de base, corte de dirección, corte de caída y rebaje del tocón.

Subsistema Desrame y Trozado: Este proceso considera dos actividades; la primera es el desrame que consiste en eliminar la totalidad de ramas que posee el fuste completo. La segunda actividad es el trozado, que constituye la corta del fuste a diferentes medidas para maximizar el aprovechamiento maderable de cada árbol, diferenciado según su importancia económica para el productor en: (i) madera aserrada de 3,2 $\mathrm{m}$ de largo, (ii) postes de 2,2 $\mathrm{m}$ de largo y (iii) leña de 1,0 m de largo. En este proceso el operador forestal especializado es quien decidió la clasificación y corte del fuste, en base a la calidad de las trozas. En esta etapa se registraron las variables largo fustal, diámetro menor de trozos maderables y numero de trozos y postes.

Subsistema Ordenación de Productos y Residuos del Raleo: Etapa que consistió en el arrumado de los productos menores (leña) obtenidos del raleo, ordenados al interior de la plantación en rumas de $1,0 \mathrm{~m}$ de altura. En esta etapa también se desarrolló la ordenación de residuos que provienen del raleo. Estos residuos quedaron ordenados al interior y en los bordes del rodal en fajas de baja altura, orientadas de forma perpendicular a las curvas de nivel. Ambas actividades fueron realizadas por dos jornales.

Subsistema Poda y Ordenamiento de Desechos: Corresponde a la eliminación de las ramas inferiores de los árboles remanentes del área de estudio. Esta faena se desarrolló por un técnico especializado, al cual se le entregó la instrucción de podar la totalidad de árboles remanentes del rodal en estudio. La poda se realizó hasta los 6,0 $\mathrm{m}$ desde la base del árbol, correspondiente al $4^{\circ} \circ 5^{\circ}$ verticilo. La herramienta utilizada para la actividad fue tijerón neozelandés y escalera de poda. Los residuos de esta actividad fueron dispuestos en las mismas fajas de las ramas acopiadas en la etapa de ordenamiento de productos y desechos del raleo.

Subsistema Madereo y Acopio: Esta etapa consiste en la extracción de los productos de mayor valor comercial para el propietario (postes y trozos) desde el interior del rodal hasta un lugar de acopio ubicado a una distancia promedio de $70 \mathrm{~m}$ (ciclo completo $=140$ $\mathrm{m})$. El madereo y acopio fue realizado por un operador utilizando una yunta de bueyes. 
Para cada actividad principal del sistema de cosecha, se registraron los tiempos del operador y variables individuales de los árboles, con ello se construyeron funciones lineales de las etapas de tiempo de volteo, desrame y trozado que pudiera servir de estimadores en futuras intervenciones. En resumen, el sistema de explotación forestal estuvo compuesto por una cuadrilla de 4 trabajadores con las siguientes funciones. 1 personal técnico que registró el tiempo de cada actividad, 1 personal jornal encargado de las labores de volteo, desrame, trozado y ordenación de residuos. 1 personal jornal especializado encargado de la poda. 1 personal jornal encargado del madereo con bueyes.

\section{Estudio de Tiempo y Rendimiento}

El estudio de tiempos es una técnica para cuantificar una tarea determinada con la mayor exactitud posible, partiendo de un número limitado de observaciones. El sentido de este trabajo es poder estimar productividad y rendimiento en una faena de raleo tardío de una plantación de pino ponderosa de 18 años de edad. Para ello, se utilizó un método de explotación tradicional que es aplicable a la realidad de los pequeños productores de la Región de Aysén.

Para la determinación del tiempo empleado en cada etapa, se ha utilizado una adecuación simple del método Vuelta a Cero o tiempo de retorno (Currie, 1979; Carey et al., 2006), el cual consiste en cronometrar cada etapa del sistema de explotación forestal y registrar la información en una planilla diseñada previamente para ser implementada en terreno, de acuerdo con los requerimientos del estudio. Las etapas del estudio de tiempos se describen en el Cuadro $N^{\circ} 1$.

\section{Cuadro $\mathrm{N}^{\circ} 1$ \\ DESCRIPCIÓN DE LAS ETAPAS EMPLEADAS EN LA EVALUACIÓN DEL ESTUDIO DE TIEMPO Y PRODUCTIVIDAD}

\begin{tabular}{|c|c|}
\hline Etapas & Descripción \\
\hline Volteo & $\begin{array}{l}\text { Corresponde al tiempo destinado entre la corta del árbol hasta el momento que cae al } \\
\text { suelo. Si el árbol queda estribado el tiempo es detenido y la actividad finaliza. }\end{array}$ \\
\hline Desestribado & $\begin{array}{l}\text { Corresponde al tiempo ocupado en "desestribar" un árbol, el cual luego de la } \\
\text { realización del corte de caída en la etapa de "volteo" no ha podido llegar al suelo de } \\
\text { forma natural quedando enganchado o en contacto con otro individuo, impidiendo que } \\
\text { se pueda desarrollar la siguiente etapa de desrame y trozado. }\end{array}$ \\
\hline Desrame & $\begin{array}{l}\text { Corresponde al tiempo destinado en quitar la totalidad de ramas al árbol, sin } \\
\text { considerar la ordenación de ramas. }\end{array}$ \\
\hline Trozado & $\begin{array}{l}\text { Corresponde al tiempo destinado en trozar el fuste completo hasta un diámetro } \\
\text { mínimo de utilización de } 10 \mathrm{~cm} \text {. El trozado se inicia desde la base del fuste } \\
\text { seleccionando trozos }(3,2 \mathrm{~m} \text { de largo), postes }(2,2 \mathrm{~m} \text { de largo }) \text { y finalmente leña }(1,0 \mathrm{~m} \\
\text { de largo). }\end{array}$ \\
\hline $\begin{array}{l}\text { Ordenamiento de } \\
\text { desechos del raleo y } \\
\text { apilado de leña }\end{array}$ & $\begin{array}{l}\text { Tiempo destinado a realizar labores de ordenación de todos los residuos derivados del } \\
\text { raleo. Estos residuos son ordenados al interior del rodal en fajas de baja altura. } \\
\text { También considera el apilado de leña al interior del rodal. }\end{array}$ \\
\hline $\begin{array}{l}\text { Poda y ordenamiento } \\
\text { de desechos de } \\
\text { poda }\end{array}$ & $\begin{array}{l}\text { Tiempo destinado a la poda de todos los árboles remanentes del rodal. Además se } \\
\text { consideró la actividad de ordenación de los desechos de poda en esta etapa. }\end{array}$ \\
\hline Madereo y acopio & $\begin{array}{l}\text { Corresponde al tiempo destinado al traslado de trozos desde el interior del rodal hacia } \\
\text { un área de acopio (distancia media de } 70 \mathrm{~m} \text { ). En esta etapa se consideró un madereo } \\
\text { tradicional a través de bueyes. }\end{array}$ \\
\hline $\begin{array}{l}\text { Tiempos } \\
\text { complementarios }\end{array}$ & $\begin{array}{l}\text { Corresponde a todos los tiempos que no forman parte del ciclo productivo de la faena } \\
\text { forestal. Se ha querido simplificar este concepto conteniendo en él todos aquellos } \\
\text { tiempos que son complementarios a la faenas forestal propiamente tal, tales como } \\
\text { tiempos de recarga de combustible y lubricantes de la motosierra, afilado de cadena, } \\
\text { limpieza del equipo, descansos del operador, fallas del equipo, etc. }\end{array}$ \\
\hline
\end{tabular}


Para determinar volumen por productos maderables (trozos) se utilizaron los datos obtenidos al momento de la etapa de trozado, midiendo el diámetro de la cara menor y el largo total de cada trozo, para posteriormente cubicarlo mediante JAS, Japanisse Standart Agriculture (Ecuación $\mathrm{N}^{\circ}$ 2). La cuantificación de otros productos (postes y leña) fue a través de medición directa en terreno. Lo anterior, permitió estimar el volumen en trozo ( $\left.\mathrm{m}^{3} / \mathrm{trozo}\right)$; volumen de leña ( $\mathrm{m}^{3} \mathrm{st} / \mathrm{ha}$ ) y numero de postes (unidades/ha).

$$
\begin{array}{rl}
V=D^{2 *}{ }^{*} & * 1 / 10.000 \\
\text { Donde: } & V=\text { Volumen }\left(\mathrm{m}^{3}\right) \\
& \mathrm{D}=\text { Diámetro de la cara menor de la troza }(\mathrm{cm}) . \\
& \mathrm{L}=\text { Largo nominal de la troza }(\mathrm{m})
\end{array}
$$

En términos generales, el concepto de productividad se define como la relación entre la cantidad de bienes producidos y la cantidad de recursos utilizados; se la puede calcular relacionando la cantidad de trabajo efectuado por unidad de tiempo (Nájera Luna, 2010). En este estudio la productividad se calculó dividiendo el resultado obtenido del raleo (en cantidad de árboles cortados y volumen apeado) por el tiempo operativo total utilizado; fue expresada en árb/h y en $\mathrm{m}^{3} / \mathrm{h}$ (de Suoza et al., 2004). Luego se estimó la productividad diaria (PD), proyectando la cantidad de metros cúbicos que se puede ralear por jornada de trabajo.

\section{Estudio de Costos}

Para determinar los costos de la faena de raleo se consideró lo siguiente:

Determinación de actividades y sub actividades consideradas dentro del raleo.

Determinación de costos por ítem, para ello se estimó el costo de la mano de obra, alimentación, herramientas e insumos.

Por tratarse este de un trabajo que pretende dar las pautas para un posible instrumento de fomento por parte del Estado, se determinó un escenario en función de la realidad de la Región de Aysén denominado "óptimo" que considera todos los costos y actividades en los que el propietario o un tercero incurriría para la realización de la faena forestal.

\section{Mano de Obra}

Para la estimación de los costos del ítem mano de obra se consideraron cuatro actividades:

Faena de raleo: Considera las labores de volteo, trozado y desrame de árboles, utilizando la unidad de medida jornadas (jor/día) para la estimación de los costos. El valor unitario de cada jornada considera que el trabajador utilice su motosierra personal.

Ordenamiento de desechos del raleo y apilado de leña: Considera dos personas para ordenar los residuos productos del raleo (biomasa obtenida de la copa principalmente) y apilado de leña al interior del rodal.

Poda y ordenación de desechos: Corresponde a la eliminación de las ramas laterales de cada árbol residual junto con la ordenación de desechos producto de esta faena. Esta labor fue desarrollada por un operario forestal, utilizando tijerón de poda neozelandés y escalera para desarrollar una faena de poda que alcanza hasta el $4^{\circ} \circ 5^{\circ}$ verticilo de cada árbol.

Madereo y acopio: Esta labor la desarrolla un trabajador forestal con sus bueyes y aperos y corresponde a la acción de extraer del bosque los productos mayores obtenidos de este manejo (postes y varas) y dejarlos ordenados en un sector de acopio para ser utilizado en mejoras prediales o disponibles para ser comercializados. 


\section{- Otros}

El ítem otros considera la estimación de costos de los elementos de protección personal, la alimentación, insumos y herramientas utilizadas directamente en la faena forestal. Para los costos de cada subítem se consideraron valores de mercado regional.

\section{RESULTADOS}

\section{Estructura Forestal}

La intervención realizada generó la disminución de la densidad $(\mathrm{N})$ en un $41 \%$ disminuyendo de 1.360 a 800 árb/ha (Cuadro $\mathrm{N}^{\circ} 2$ 2), generando un espaciamiento medio de $3,5 \mathrm{~m}$ entre cada árbol remanente. De la misma manera se disminuyó el área basal de 53,3 a $34 \mathrm{~m}^{2} / \mathrm{ha}$ lo que representó una intervención de un $36 \%$ del área basal $(A B)$.

\section{Cuadro $\mathrm{N}^{\circ} 2$}

PROMEDIOS Y DESVÍOS DE LAS PRINCIPALES VARIABLES DE LA ESTRUCTURA ANTES Y DESPUÉS DEL RALEO

\begin{tabular}{|l|c|c|c|c|}
\hline \multicolumn{1}{|c|}{ Condición } & $\begin{array}{c}\mathbf{N} \\
(\mathbf{a r b} / \mathbf{h a})\end{array}$ & $\begin{array}{c}\mathbf{A B} \\
\left(\mathbf{m}^{2} / \mathbf{h a}\right)\end{array}$ & $\begin{array}{c}\mathbf{D C M} \\
\mathbf{( c m})\end{array}$ & $\begin{array}{c}\text { HD } \\
(\mathbf{m})\end{array}$ \\
\hline Antes raleo & $1.360 \pm 105,83$ & $53,26 \pm 1,68$ & $22,37 \pm 1,17$ & $10,80 \pm 0,20$ \\
\hline Después raleo & $800 \pm 105,83$ & $34,03 \pm 2,42$ & $23,36 \pm 1,59$ & $11,83 \pm 0,76$ \\
\hline
\end{tabular}

N: Densidad; AB: Area Basal; DCM: Diámetro Cuadrático Medio; HD: Altura Dominante

El raleo por lo bajo permitió aumentos en algunos parámetros del rodal (Figura $\mathrm{N}^{\circ} 2$ ). En el caso del diámetro medio cuadrático (DMC) ocurrió una variación positiva de 22,3 a 23,3 cm mientras que para la altura media (HM) también se observó un incremento de 1,0 m.

D
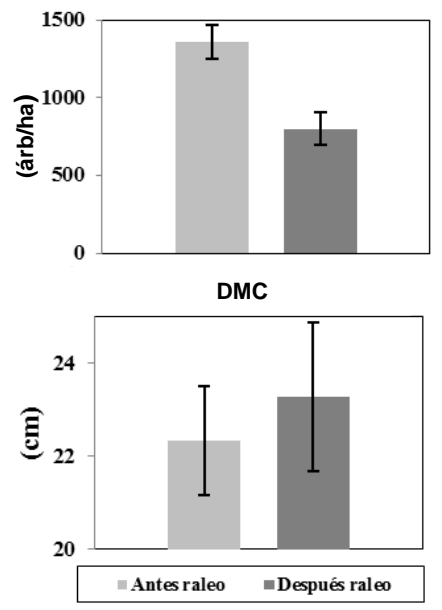

AB



HD

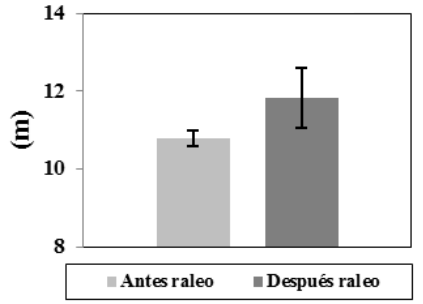

Figura $\mathrm{N}^{\circ} 2$

VARIACIÓN DE LOS PARÁMETROS DASOMÉTRICOS ANTES Y DESPUÉS DEL RALEO 
La intensidad de raleo aplicado a la plantación no hizo variar la estructura regular. Debido a la nula intervención anterior de esta plantación y lo tardío del manejo realizado, fue necesario aplicar una combinación de raleo por lo bajo y por los alto (Figura $N^{\circ} 3$ ).
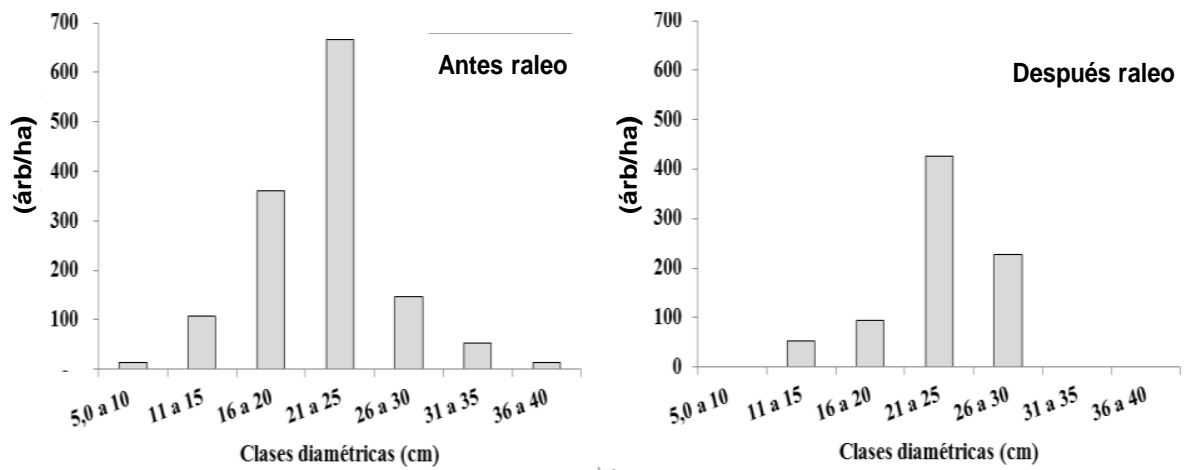

Figura $\mathrm{N}^{\circ} 3$

DISTRIBUCIÓN DIAMÉTRICA

\section{Estudio de Tiempo}

Este estudio permitió describir los tiempos de cada una de las cuatro grandes actividades que componen la faena forestal; raleo, poda, ordenamiento de desechos y madereo. La actividad de raleo estuvo compuesta por cuatro sub actividades, que correspondieron al volteo, desestribado, desrame y trozado.

En cada una de ellas se determinó el tiempo promedio de cada subactividad con el fin de determinar cuáles de ellas demandan mayor tiempo (Figura $N^{\circ} 4$ ).
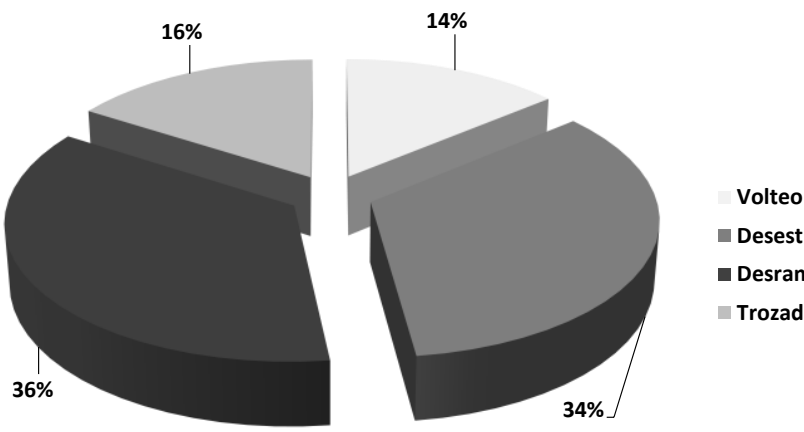

Desestribado

- Desrame

Trozado

Figura $N^{\circ} 4$

TIEMPO DESTINADO A CADA SUB ACTIVIDAD

Los procesos que demandan mayor tiempo en la actividad de raleo son el desrame, desestribado, trozado y volteo, con estimaciones porcentuales de $36 \%, 34 \%, 16 \%$ y $14 \%$, respectivamente.

Del mismo modo se determinaron los tiempos de todas las actividades de la faena forestal, 
incluidos los tiempos complementarios, para determinar aquellas actividades que demandan mayor y menor tiempo.

Las actividades que demandaron mayor tiempo en la faena de raleo fueron la ordenación de desechos y el madereo, cada una con un total de 4,0 jor/ha (un $26 \%$ del total), seguidas por el raleo y poda con un tiempo de ejecución de 3,8 y 3,0 jor/ha, respectivamente (Figura № 5).

Mientras que la actividad que demandó menor periodo de ejecución fue el tiempo complementario con $1,0 \mathrm{jor} / \mathrm{ha}(6,8 \mathrm{~h} / \mathrm{ha})$. El tiempo total destinado a la faena forestal fue aproximadamente $125 \mathrm{~h} / \mathrm{ha}$, esto es un total de $15,6 \mathrm{jor} / \mathrm{ha}$.

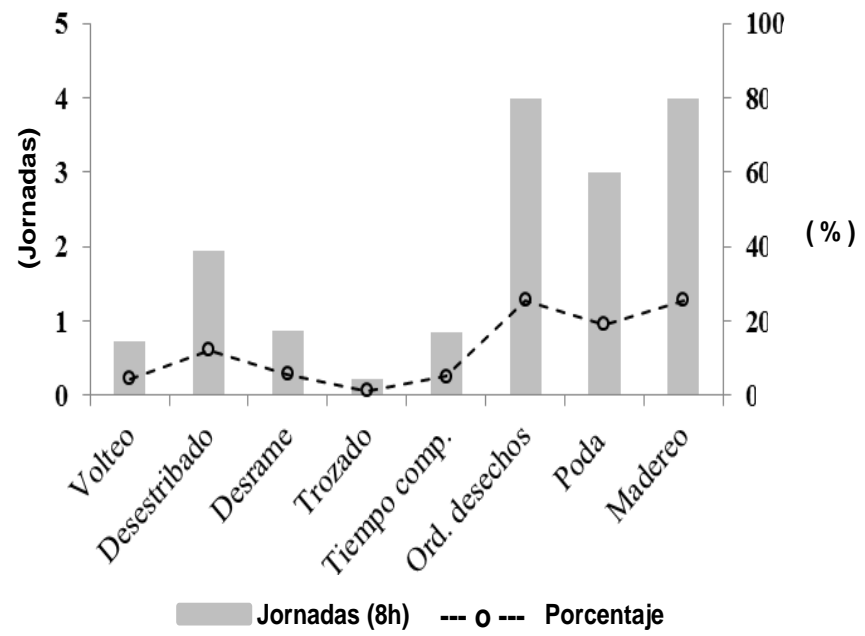

JORNADAS DE TRABAJO Y PORCENTAJE DESTINADO A CADA ACTIVIDAD DE LA FAENA

En la Figura $N^{\circ} 6$ se muestra el análisis de la relación entre el DAP y los tiempos de tres de las cuatro actividades principales de la etapa de raleo (volteo, desrame y trozado).

En cada actividad se registraron los tiempos que tardó el operador para cada individuo y para cada una se desarrolló una ecuación lineal que puede servir de estimador de las prácticas implementadas.

El tiempo de volteo no fue explicado de buena forma por la ecuación lineal $\left(R^{2}=0,3\right)$, esto se podría relacionar a diferentes externalidades que ocurren en esta actividad, dada la alta densidad del rodal que provoca que los fustes queden estribados al momento de realizar el volteo.

En el caso de los tiempos destinados a las actividades de desrame y de trozado las ecuaciones los explican en un $70 \%$ y un $55 \%$, respectivamente. 

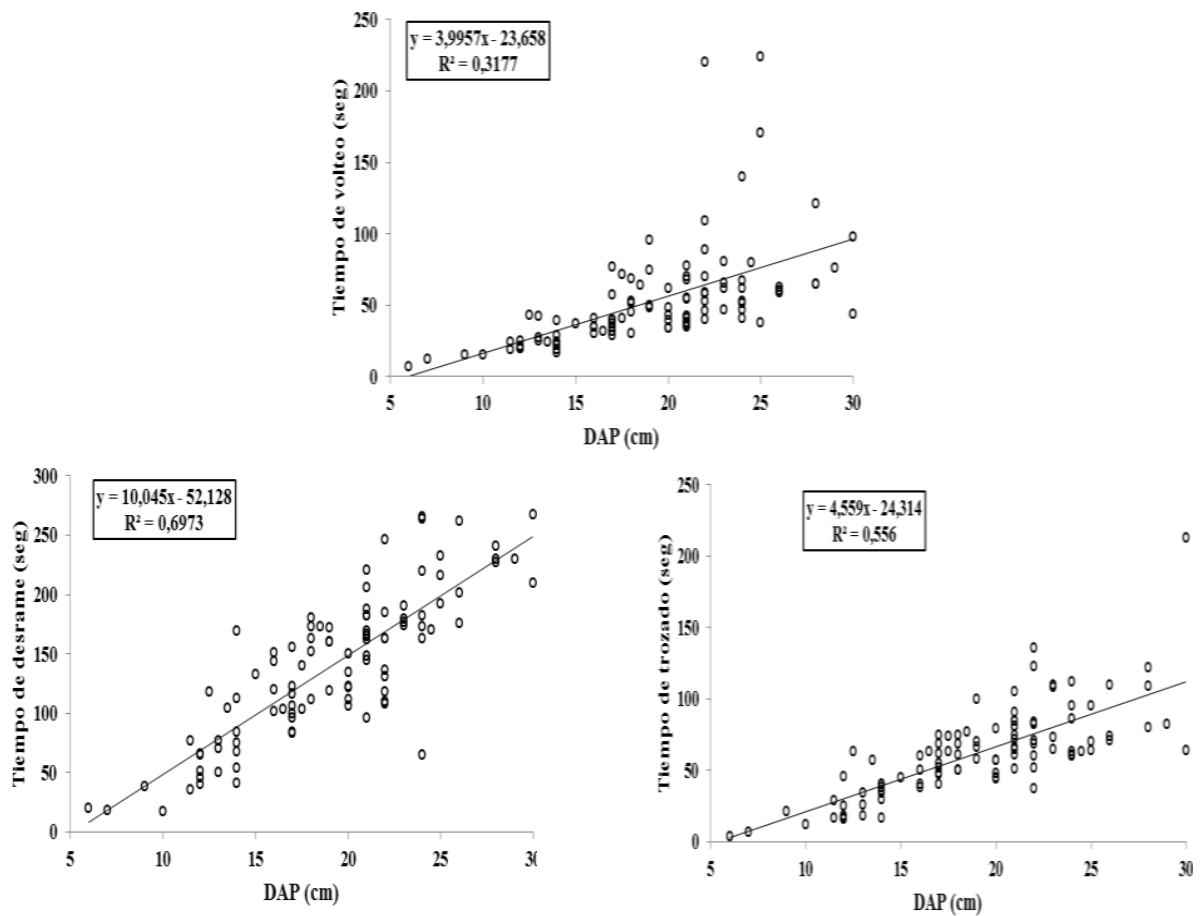

Figura $\mathrm{N}^{\circ} 6$

RELACIÓN DEL DIÁMETRO A LA ALTURA DEL PECHO (DAP) CON LOS TIEMPOS

DE LAS SUB ACTIVIDADES DE VOLTEO, DESRAME Y TROZADO

\section{Estudio de Costos}

En el Cuadro $\mathrm{N}^{\circ} 3$ se muestra la estructura de costos de la faena, el costo total considerando valores de mano de obra, insumos y herramientas fue de $\$ 963.761 /$ ha $(20,38$ UTM/ha).

El mayor costo fue por concepto de mano de obra, que representó un $75 \%$ del costo total y las actividades de mayor costo fueron el raleo, la ordenación de desechos, el madereo y la poda, representando el $43 \%, 23 \%, 20 \%$ y $14 \%$ del total de mano de obra, respectivamente.

\section{Cuadro 3}

\section{ESTRUCTURA DE COSTOS}

\begin{tabular}{|c|c|c|c|c|c|c|}
\hline Labores & Unidad & $\begin{array}{c}\text { Personas } \\
\text { (№) }\end{array}$ & $\begin{array}{c}\text { Jornadas } \\
\qquad\left(\mathrm{N}^{\circ}\right)\end{array}$ & $\begin{array}{c}\text { Unidad } \\
\text { (\$) }\end{array}$ & $\begin{array}{l}\text { Valor } \\
(\$ / \text { ha) }\end{array}$ & $\begin{array}{l}\text { Valor }^{\star \star} \\
\text { (UTM/ha) }\end{array}$ \\
\hline \multirow{2}{*}{ Raleo* } & Jornadas & 1 & 4,5 & 50.000 & 225.000 & 4,76 \\
\hline & Jornadas & 1 & 4,5 & 20.000 & 90.000 & 1,90 \\
\hline Ordenamiento de desechos y apilado de leña & Jornadas & 2 & 4,1 & 20.000 & 164.000 & 3,47 \\
\hline Poda y ordenación & Jornadas & 1 & 3,0 & 35.000 & 105.000 & 2,22 \\
\hline Madereo y acopio & Jornadas & 1 & 4,1 & 35.000 & 143.500 & 3,03 \\
\hline \multicolumn{5}{|c|}{ Subtotal Mano de Obra (A) } & 727.500 & 15,38 \\
\hline
\end{tabular}


Cuadro $N^{\circ} 3$ continuación

\begin{tabular}{|c|c|c|c|}
\hline Labores & Detalle & $\begin{array}{l}\text { Valor } \\
\text { (\$/ha) }\end{array}$ & $\begin{array}{c}\text { Valor } \\
\text { (UTM/ha) }\end{array}$ \\
\hline \multirow{4}{*}{ Raleo } & Elementos protección personal & 4.315 & 0,09 \\
\hline & Alimentación & 31.500 & 0,67 \\
\hline & Insumos & 38.160 & 0,81 \\
\hline & Herramientas & 9.902 & 0,21 \\
\hline \multirow{4}{*}{ Ordenamiento de desechos y apilado de leña } & Elementos protección personal & 3.186 & 0,07 \\
\hline & Alimentación & 57.400 & 1,21 \\
\hline & Insumos & 0 & 0 \\
\hline & Herramientas & 559 & 0,01 \\
\hline \multirow{4}{*}{ Poda y ordenación } & Elementos protección personal & 609 & 0,01 \\
\hline & Alimentación & 10.500 & 0,22 \\
\hline & Insumos & 0 & 0 \\
\hline & Herramientas & 2.225 & 0,05 \\
\hline \multirow{4}{*}{ Madereo y acopio } & Elementos protección personal & 832 & 0,02 \\
\hline & Alimentación & 14.350 & 0,3 \\
\hline & Insumos & 0 & 0 \\
\hline & Herramientas & 62.723 & 1,33 \\
\hline \multicolumn{2}{|r|}{ Subtotal insumos y herramientas (B) } & 236.261 & 5,00 \\
\hline & COSTO TOTAL $(A+B)$ & 963.761 & 20,38 \\
\hline
\end{tabular}

* Valores mano de obra: Operador motosierra $\$ 50.000 /$ jornada; operador jornal $\$ 20.000 /$ jornada; operador poda y madereo $\$ 35.000 /$ jornada.

** Valor UTM correspondiente al mes de marzo de 2018.

\section{Rendimiento de Productos}

De los árboles raleados fue posible obtener 3 tipos de productos que quedan disponibles para el uso del propietario. Estos productos valorizados y llevados a un valor por hectárea son detallados en el Cuadro $\mathrm{N}^{\circ} 4$.

\section{Cuadro 4 \\ PRODUCCIÓN}

\begin{tabular}{|c|c|c|c|c|c|}
\hline \multirow{2}{*}{ Producto } & \multirow{2}{*}{ Unidad } & \multirow{2}{*}{$\begin{array}{l}\text { Cantidad } \\
\text { (Unidades) }\end{array}$} & \multirow{2}{*}{$\begin{array}{c}\text { Valor* } \\
(\$)\end{array}$} & \multicolumn{2}{|c|}{ Total } \\
\hline & & & & (\$/ha) & $(\text { UTM/ha })^{\star \star}$ \\
\hline Madera aserrada & Pulgada pinera & 246 & 2.500 & 615.000 & 13,00 \\
\hline Postes & Unidad & 464 & 1.000 & 464.000 & 9,81 \\
\hline Leña & Metro Estéreo & 40 & 8.000 & 320.000 & 6,77 \\
\hline & & & Total ingresos & 1.399 .000 & 29,58 \\
\hline
\end{tabular}

*Valor comercial: Precios referenciales de los productos puestos en el predio según mercado local.

** Valor UTM correspondiente al mes de marzo de 2018.

En las condiciones particulares de la plantación de pino ponderosa estudiada los ingresos producto de la intervención fueron de $\$ 1.399 .000$ /ha. Los productos que generaron los retornos al productor fueron la madera aserrada, postes y leñas, con un $44 \%, 33 \%$ y $23 \%$ del total, respectivamente. 


\section{DISCUSION}

A inicios de la década de los 70 y con el propósito de recuperar suelos erosionados y en proceso de degradación, se llevó a cabo un plan de forestación con coníferas exóticas en la Región de Aysén. Sin embargo, aún no se dispone de información suficiente acerca de la rentabilidad esperable de esta inversión que oriente decisiones públicas o privadas. Las acciones silvícolas practicadas en el área de estudio corresponden a la primera intervención de carácter "tardío" implementadas en un rodal de pino ponderosa de 18 años de edad. Con estas actividades de manejo en plantaciones forestales se busca poner en producción sitios en desuso y de poco valor.

La aplicación de cortas intermedias además de fomentar el crecimiento y productividad maderera, provoca condiciones que estimulan el crecimiento de la pradera aumentando la base forrajera del predio y de esta forma aseguran producción diversificada (Peri et al., 2009). La importancia de los sistemas silvopastorales está dada por los múltiples beneficios que pueden generar a los productores, al integrar la actividad ganadera con la obtención de productos madereros provenientes del manejo del bosque.

De esta forma, se transforman en un sistema continuo que otorga ingresos anuales derivados de prácticas pecuarias y productos intermedios del manejo del bosque (Salinas et al., 2017). En este sentido, las prácticas de raleos en plantaciones forestales podrían constituirse en intervenciones de características sinérgicas, en las que se mejora el crecimiento de los árboles remanentes y a la vez la producción de forraje y el uso por parte de los animales de la cubierta forestal para la protección en invierno, integrado esto con la conservación de la biodiversidad, el paisaje y la calidad de vida de las comunidades (Peri y Ormaechea, 2009).

Una pregunta recurrente entre autoridades de gobierno, tanto nacional como local, es si existen antecedentes para probar la contribución de los Sistemas Agroforestales a la economía rural y a su sustentabilidad en Chile, para así promover su uso. Existen diversos estudios, tanto de autores chilenos como extranjeros, que valoran el aporte de los árboles y arbustos en sistemas agroforestales en la protección del medio natural, donde se ha documentado que pueden reducir la erosión y modificar algunos aspectos climáticos del entorno en beneficio de la agricultura y ganadería, si estos se usan en forma integrada con algún ordenamiento espacial.

Algunos de los beneficios que se han identificado para la integración de especies arbóreas y animales bajo sistemas silvopastorales en predios ganaderos, agrícolas de secano y/o forestales tradicionales, son aprovechar la protección que puede ofrecer el árbol, tanto a los animales como a la pradera y cultivos, frente a condiciones climáticas adversas (Sotomayor, 1989); mejorar la belleza escénica del predio y el valor de la propiedad (Sotomayor y Cabrera, 2006; Sotomayor 2009), y proteger y mejorar los suelos (Murgeitio, 2009), entre otros. Estos antecedentes son los que han permitido promover el uso de sistemas agroforestales en Chile.

Según Carey et al. (2006) un sistema de cosecha forestal en plantaciones de Eucalyptus $s p$. de corta rotación se caracteriza por la alta ocupación en mano de obra y una baja escala de producción. Por el contrario, el manejo de las plantaciones de la Región de Aysén ocupa baja cantidad de mano de obra, tal como se describe en el presente estudio, donde solo se utiliza una cuadrilla compuesta por dos personas y una yunta de bueyes.

El sistema de madereo se realizó a favor de la pendiente, con distanciamientos medios correspondientes a $70 \mathrm{~m}$. Tal como indica Silverades y Segerström (1983), este tipo de madereo a favor de la pendiente, con distancia de madereo promedio de $50 \mathrm{~m}$, favorece la faena con bueyes.

Este tipo de manejo se desarrolla en condiciones normales de un propietario de la Región de Aysén, donde la actividad forestal se ha ganado un espacio dentro del quehacer tradicional rural, pues se ha demostrado que este tipo de acciones genera ingresos y aportan de manera significativa a la economía familiar campesina.

Por años se creía que el manejar plantaciones forestales en la Región de Aysén no generaba utilidades aplicando las primeras cortas intermedias, sin embargo queda demostrado que 
las utilidades podrían incluso verse aumentadas al poner a disposición productos forestales primarios tales como leña, postes y trozos para la producción de madera aserrada, productos respecto de los cuales no se conocen a cabalidad sus características técnicas.

La madera aserrada y los postes de pino ponderosa han venido ganando un espacio al interior del mercado local, posicionándose como una alternativa económicamente viable para acciones de construcción y reparación de infraestructura primaria al interior de los mismos predios donde se producen las intervenciones. Además, la leña de coníferas está siendo adquirida y valorada por los consumidores, transformándose en una alternativa para solucionar problemas sociales de la población, en especial en época invernal.

Actualmente no existe una política de estado que financie el manejo de plantaciones forestales en Chile. Desde la expiración del D.L. № 701 en el año 2012, no se ha incentivado el manejo en este tipo de plantaciones, lo que se observa particularmente en la Región de Aysén donde se encuentran plantaciones sin manejo, principalmente de pequeños propietarios, que no aportan ingresos a la economía familiar campesina, pues sus tasas de crecimiento están muy por debajo de lo que podrían estar con un manejo oportuno.

Estos terrenos podrían estar siendo utilizados bajo un sistema silvopastoral, contribuyendo con productos forestales primarios por concepto del árbol y producción agrícola y/o pecuaria de la pradera natural. Mientras el estado no asuma un rol protagónico en estas materias, estos terrenos seguirán siendo subutilizados, desincentivando a los dueños de bosque a seguir forestando.

\section{CONCLUSIONES}

Los resultados de este estudio de tiempo y rendimiento permiten por primera vez en la Región de Aysén determinar los productos madereros obtenidos en la faena de raleo y, a la vez, conocer el costo de manejo de una plantación juvenil de pino ponderosa. Con esta información, fidedigna de un trabajo de campo, los organismos del Estado tendrían la base para implementar instrumentos de fomento al manejo forestal de estas plantaciones.

El sistema descrito en el estudio es una opción aplicable en faenas forestales de pequeña escala en la Región de Aysén incluso en invierno cuando la ocupación de mano de obra es escasa.

La información presentada en este estudio es de interés para productores y técnicos del rubro forestal y muestra la utilidad real que genera el manejo de plantaciones forestales en la región. Producto de la intervención silvícola aplicada a la plantación de pino ponderosa de 18 años de edad, fue posible obtener una utilidad de $\$ 435.247$ /ha, la que podría convertirse en un atractivo para reactivar el manejo de las plantaciones de la Región de Aysén.

El margen de ganancia indicado para los productores podría verse facilitado por un instrumento de fomento estatal que los apoye en las primeras fases del manejo.

El desarrollo de sistemas silvopastorales en plantaciones forestales de la Región de Aysén podría constituir una alternativa productiva sustentable, que además ofrece la posibilidad de recuperar ecosistemas degradados.

Con los resultados expuestos en este trabajo se fundamenta técnicamente la posibilidad de generar un proceso que incentive el manejo de plantaciones forestales en la región y su conversión a sistemas silvopastorales, con los beneficios para los propietarios de mejoramiento de los ingresos del grupo familiar, aumento de la productividad de los predios, control de la erosión y provisión de material energético para sus hogares.

Es entonces un desafío para legisladores e instituciones de fomento generar un instrumento específico que incentive el manejo de plantaciones, permitiendo transformarlas en áreas productivas de uso múltiple y constituyendo un valioso aporte al desarrollo rural en una región extrema del país. 


\section{REFERENCIAS}

Carey, B.; Figueroa, S. y Valenzuela, C., 2006. Evaluación técnica de un sistema tradicional de cosecha en plantaciones de Eucalyptus globulus de corta rotación en Valdivia, Chile. Bosque (Valdivia), 27(3), 272-276.

Currie, R., 1979. Análisis y medición del trabajo. México D.F., México. Diana. 348 p.

De Souza, A. P.; Minette, L. J.; Moreira, F. M. T.; Machado, C. C. y Silva, K. R., 2004. Análise do desempenho da máquina slingshot em subsistemas de colheita em florestas de eucalipto. Revista Brasileira de Engenharia Agrícola e Ambiental, 8(2-3): 316-320.

Gonda, H., 2001. Manejo de Pino Ponderosa. CIEFAP- Patagonia Forestal, Año VII, N 3, pp7-10.

Hepp, C. y Stolpe, N. B., 2014. Caracterización y propiedades de los suelos de la Patagonia occidental (Aysén). Instituto de Investigaciones Agropecuarias, Centro de Investigación INIA Tamel Aike, Coyhaique, AysénPatagonia, Chile. $160 \mathrm{pp}$.

INE, 1997. VI Censo Nacional Agropecuario. Santiago, Chile.

INFOR, 2018. Anuario forestal 2018. Instituto Forestal, Chile. Boletín Estadístico № 163. Santiago, Chile. 188 pp.

Quintanilla, V., 2008. Perturbaciones a la vegetación nativa por grandes fuegos de 50 años atrás, en bosques nordpatagónicos. Caso de estudio en Chile Meridional. Anales de Geografía 28(1): 85-104.

Murgeitio, E., 2009. Aspectos relacionados con la sustentabilidad social y ambiental de los sistemas silvopastoriles en América tropical. En: Actas del 1er Congreso Nacional de Sistemas Silvopastoriles. Posadas, Misiones Argentina. Ed. Instituto Nacional de Tecnología Agropecuaria, Buenos Aires, Argentina. Pp. 66-69.

Nájera Luna, J. A., 2010. Evaluación del proceso productivo maderable en la región de El Salto, Durango, México [Tesis doctoral]. Nuevo León: Facultad de Ciencias Forestales. Universidad Autónoma de Nueva León. $198 p$.

Peri, P. y Ormaechea, S., 2009. Relevamiento de los bosques nativos de ñire (Nothofagus antarctica) en Santa Cruz: Base para su conservación y manejo. Ediciones INTA. Santa Cruz, Argentina. 89 p.

Peri, P. L.; Hansen, N.; Rusch, V.; Tejera, L.; Monelos, L.; Fertig, M.; Bahamonde, H. y Sarasola, M., 2009. Pautas de manejo de sistemas silvopastoriles en bosques nativos de Nothofagus antarctica (ñire) en Patagonia. Actas Primer Congreso Nacional de Sistemas Silvopastoriles, pp. 151-164, Ediciones INTA. Posadas, Misiones, 14 al 16 de mayo 2009.

Salinas, J. y Acuña, B., 2013. Conversión de plantaciones forestales a sistemas silvopastorales en la Región de Aysén. Revista Ciencia e Investigación Forestal INFOR, Volumen 19 N 1.12 p.

Salinas, Jaime; Peri, Pablo L.; Hepp, Christian y Acuña, Bernardo, 2017. Sistemas Silvopastorales en Bosques de Nirre (Nothofagus antarctica (G. Forst.) Oerst.) de la Región de Aysén. Instituto Forestal, Chile. Documento de Divulgación N43. 60 p.

Silverades, R. y Segerström, G., 1983. Tecnología básica en operaciones forestales. Roma. FAO. 122 p. (Estudio FAO Montes, 36).

Sotomayor, A., 1989. Sistemas silvopastorales y su manejo. Documento Técnico № 42. Revista Chile Forestal, Diciembre 1989. CONAF. 8p.

Sotomayor, A., 2009. Sistemas silvopastorales, alternativa de producción integrada para un desarrollo sustentable de la agricultura en Chile. Ciencia e Investigación Forestal, Volumen 16 N 1: pp. 19-51.

Sotomayor, A. y Cabrera, C., 2006. Análisis de un Sistema Silvopastoral con Pinus radiata D. Don, Asociado con Ganado Ovino en la Zona Mediterránea Costera Central de Chile. En: 3er Congreso de Ciencias Forestales, organizado por la Sociedad de Ciencias Forestales de Chile. Concepción, Chile. 
